Physics

Electricity \& Magnetism fields

Okayama University

Year 1988

\title{
Computation accuracies of boundary element method and finite element method in transient eddy current analysis
}
H. Tsuboi*
M. Tanaka ${ }^{\dagger}$
T. Misaki ${ }^{\ddagger}$
T. Naito**

* Okayama University

†Okayama University

‡Okayama University

**Fuji Electric Corporate Research and Development, Limited

This paper is posted at eScholarship@OUDIR : Okayama University Digital Information Repository.

http://escholarship.lib.okayama-u.ac.jp/electricity_and_magnetism/118 
H. Tsuboi, M. Tanaka and T. Misaki

Department of Electrical and Electronic Engineering, Okayama University, Okayama 700, Japan
T. Naito

Fuji Electric Corporate Research and Development, Ltd. Hino, Tokyo 191, Japan
Abstract - In this paper, computation accuracies of boundary element method and finite element method in transient eddy current problems are compared by using a slot-embedded conductor model and a diffusion model which can be solved theoretically. The computation accuracies of vector potential or magnetic flux density by BEM and FEM were evaluated and it was clear that larger stepwidth of time can be chosen in BEM compared with FEM for the same accuracy.

\section{INTRODUCTION}

Transient eddy current analysis is one of urgent requirements in the design of electrical equipment. The finite element method (FEM) and boundary element method (BEM) are mainly used as numerical methods of transient eddy current analysis [1],[2]. Several works for transient eddy current analysis have been done[3][4], and the authors have received many invaluable suggestions from these works.

In this paper, computation accuracies of the BEM and FEM for two-dimensional transient problems are compared. In the BEM, triangular elements with constant vector potential were used for domain integrals and unknown vector potential or flux was assumed to be constant only on each boundary element. In the FEM, triangular elements with quadratically varying vector potential were used. A slot-embedded conductor model and a diffusion model which can be solved theoretically are chosen as computation model, and computation accuracies of vector potential or magnetic flux density by $B E M$ and FEM are evaluated.

\section{FORMULATION}

\section{Boundary Element Method}

Using the magnetic vector potential and the scalar potential, the differential equation to be solved is given from Maxwell's equations in twodimensiona1 problems as follows:

$$
\frac{1}{\mu \sigma} \nabla^{2} A=\frac{\partial A}{\partial t}+\frac{\partial \phi}{\partial z}
$$

where $A$ is the $z$-component of vector potential, $\phi$ is the scalar potential, $\mu$ is the permeability and $\sigma$ is the conductivity. Here, the time differentiation in Eq. (1) is approximated by the difference:

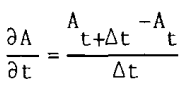

From Eq. (2), Eq. (1) is rewritten as

$$
k \nabla^{2} A_{t+\Delta t}-\frac{1}{\Delta t} A_{t+\Delta t}=\frac{1}{\Delta t} A_{t}+\frac{\partial \phi}{t+\Delta t}
$$

where $\mathrm{k}=1 /(\mathrm{H} \sigma)$.

The fundamental solution of Eq. (3), which is the Helmholtz type, is given by

$$
A_{t+\Delta t}^{*}=\frac{1}{2 \pi k} K_{0}\left(\frac{r}{\sqrt{k \Delta t}}\right)
$$

where $K_{0}$ is the modified Bessel function of the second kind of zero order. From Eq. (3), one can obtained the basic equation of BEM for the field point on the boundary surface as follows:

$$
\begin{aligned}
& \frac{1}{2} A_{i t+\Delta t}+k \int_{\Gamma} A_{t+\Delta t} \frac{\partial A_{t+\Delta t}^{*}}{\partial n} d \Gamma \\
& =k \int_{\Gamma} \frac{\partial A}{\partial n} A_{t+\Delta t}^{*} d \Gamma+\frac{1}{\Delta t} \int_{\Omega}\left(A_{t}-\frac{\partial \phi}{\partial t+\Delta t}\right) A_{t+\Delta t}^{*} d \Omega
\end{aligned}
$$

When applying the BEM for transient problems, the region to be analyzed is divided into triangular elements and the boundary surface is divided into segments which are called boundary elements. Here, vector potentials on the triangular element and boundary element are assumed both to be constant. The triangular elements are used only for domain integral appeared in $\mathrm{Eq}$. (5), and unknown vector potential or unknown magnetic flux density is defined on each boundary element. By using Eq. (5), the final simultaneous equations are set up for unknown vector potentials and magnetic flux densities on the boundary elements.

\section{Finite Element Method}

The simultaneous equations of FEM for magnetic vector potentials which satisfy $\mathrm{Eq}$. (1) are given by

$$
[S]\{A\}=\left(-\frac{\partial \phi}{\partial z}\right)(g)-[G] \frac{\partial}{\partial t}\{A\}
$$

where $\{A\}$ is the unknown vector potential, [G], [S] and (g) are defined by triangular mesh and the physical constant of material.

Approximating the time differentiation in Eq. (6) by central differences, the following equation is obtained

$$
\begin{aligned}
& \left(\frac{1}{\Delta t}[G]+\frac{1}{2}[S]\right) A_{t+\Delta t} \\
& \quad=\left(\frac{1}{\Delta t}[G]-\frac{1}{2}[S]\right) A_{t}+\frac{1}{2}\left(-\frac{\partial \phi_{t+\Delta t}}{\partial z}-\frac{\partial \phi_{t}}{\partial z}\right)\{g\}
\end{aligned}
$$

When applying the FEM, the region to be analyzed is divided into triangular elements. Then, the vector potential on the triangular element is approximated by a quadratic function. The final simultaneous equations are set up by using Eq. (7).

\section{Initial-Value Problem}

The transient eddy current problem is solved as a initial-value problem by using Eq. (5) for the BEM or Eq. (7) for the FEM. Therefore, the vector potential $\{A\}_{t+A t}$ is solved using a known vector potential $\{A\}$, which is the initial value, at each time step. At first time step, computations of the coefficients of $\mathrm{Eq}$. (5) or (7) are completed, and at each time step, some matrix calculations are done in order to obtain $(A)_{t+\Delta t}$. 


\section{COMPUTATION RESULTS}

\section{Slot-Embedded Conductor Model}

Figure 1(a) shows a slot-embedded conductor model which is solved theoretically. Because time dependence of this model is sinusoidal, evaluation of the computation accuracy was done after ten cycles of impressed potential, which is given by

$\partial \phi$

$$
\bar{\partial}^{\mathrm{t} z}=-\mathrm{V} \cos (\omega \mathrm{t}) \text {. }
$$

Eq. (8) is equivalent to current source.

Figure 2 shows the maximum difference of the value of vector potential obtained by the BEM and the FEM using triangular meshes $A$ and $B$ shown in Fig. 1(a) and Fig. 1(b). From Fig. 2, it was clear that larger stepwidth of time can be chosen in the BEM compared with the FEM for the same accuracy. In addition, Fig. 2 shows that more accurate solution can be expected by BEM using smaller triangular mesh. The variations of the vector potential in term of time are shown in Fig. 3 , and the distributions of the vector potential in the slot are shown in Fig. 4.

\section{Diffusion Mode1}

Figure 5 shows a diffusion model in which impressed magnetic flux density is a unit step function. In this model, the basic equation is obtained by replacing the vector potential A by magnetic flux density $\mathrm{B}$ in Eqs. (5) and (7).

Using the triangular meshes $C$ and $D$ shown in Fig. 5(c) and Fig. 5(d), the computation results shown in Fig. 6 and Fig. 7 were obtained. In diffusion model, the difference between computation results of BEM and those of FEM was very small.

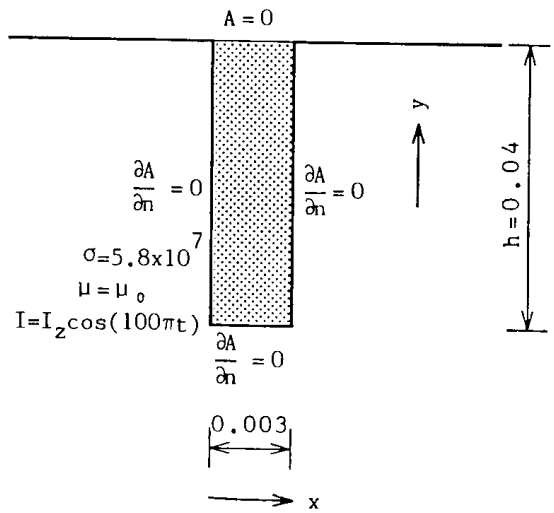

(a)

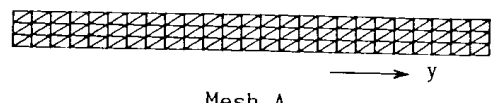

(b)

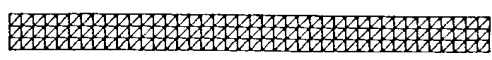

Mesh B

(c)

Fig. 1 Slot-embedded conductor model, (a) computation model, (b) mesh $A,(c)$ mesh $B$.

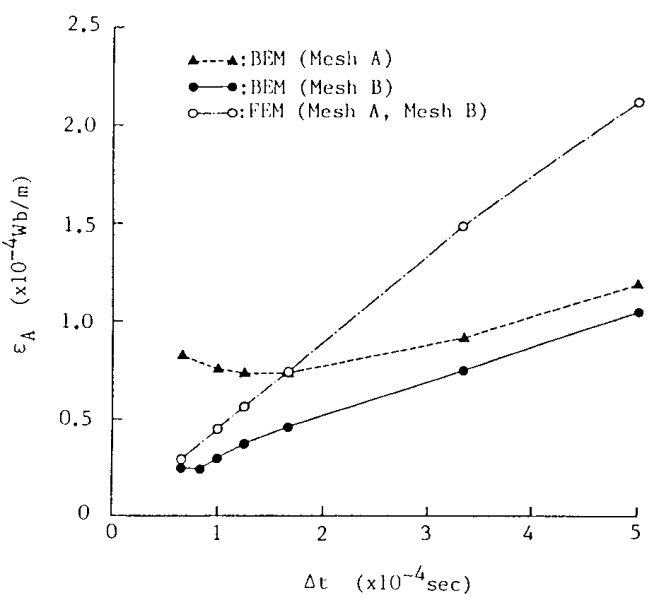

Fig. 2 Maximum difference, $\varepsilon_{A}$, between computed and theoretical values of vector potential.

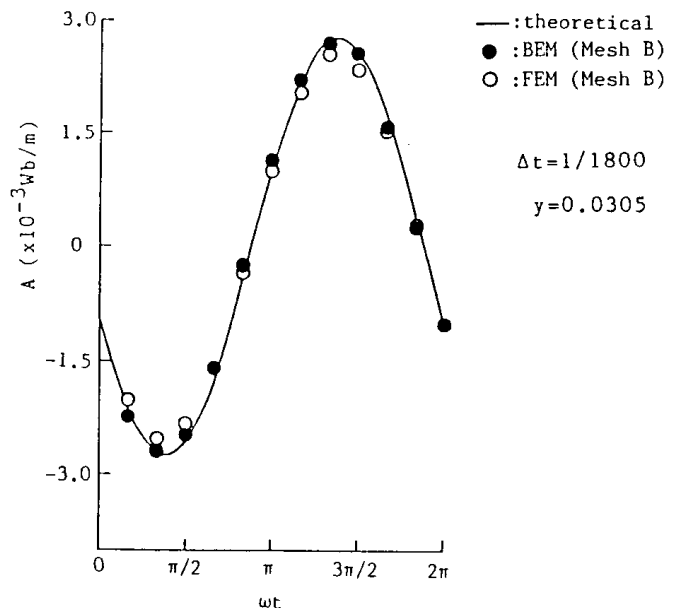

(a)

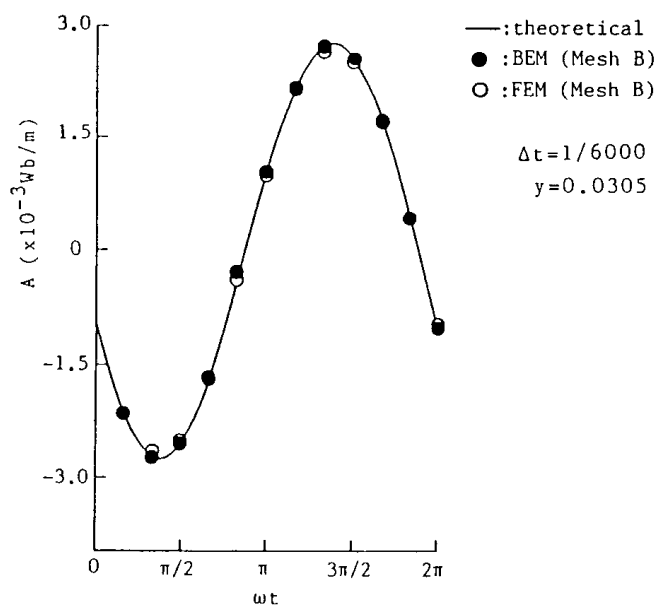

(b)

Fig. 3 Variations of the vector potential at $y=0.0305$, (a) $\Delta t=1 / 1800$, (b) $\Delta t=1 / 6000$. 


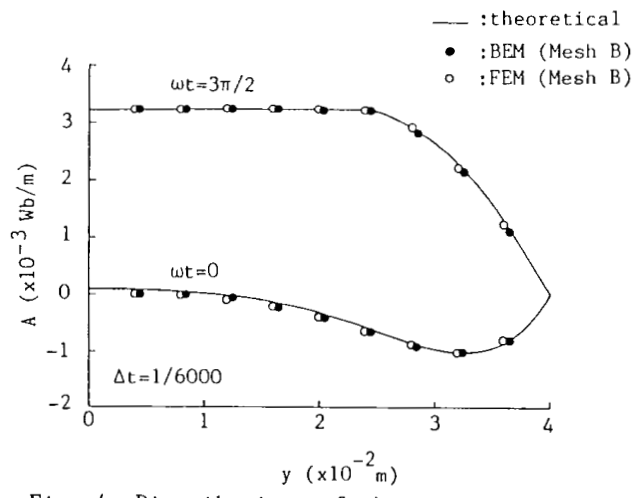

Fig. 4 Distributions of the vector potential in the slot-embedded conductor model.

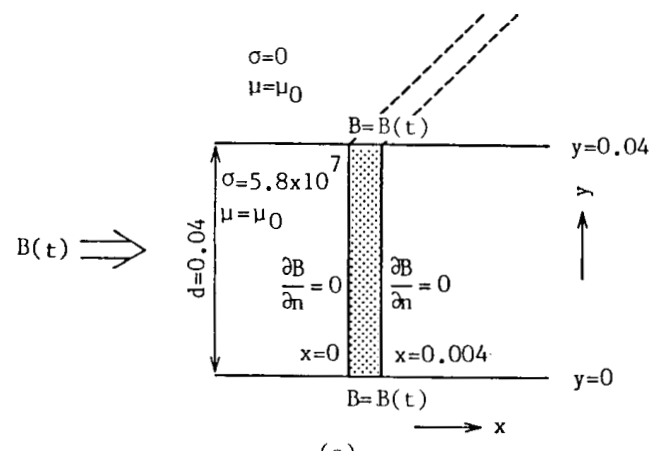

(a)

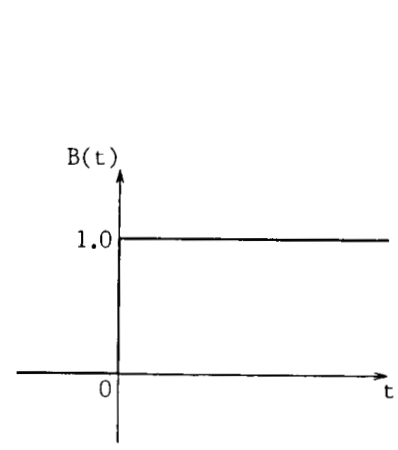

(b)

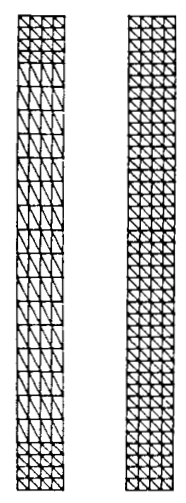

Mesh C Mesh D

(c)

(d)

Fig. 5 Diffusion mode1, (a) computation model, (b) impressed magnetic flux density, (c) mesh C, (d) mesh D.

\section{CONCLUSION}

Computation accuracies of the BEM and the FEM in the slot-embedded conductor model and the diffusion model were evaluated. As the result, it was clear that larger stepwidth of time can be chosen in the BEM compared with the FEM for the same accuracy; however the BEM and the FEM provide similar results, in the case of no current source, as the diffusion model.

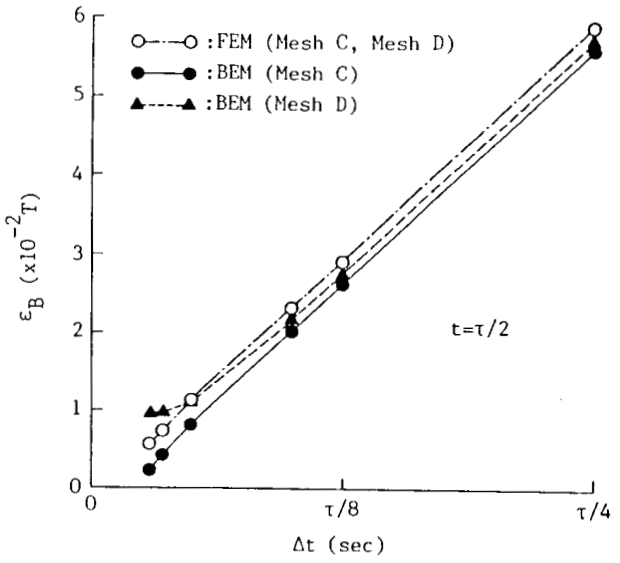

Fig. 6 Maximum difference, $\varepsilon_{B}$, between computation and theoretical values of vector potential.

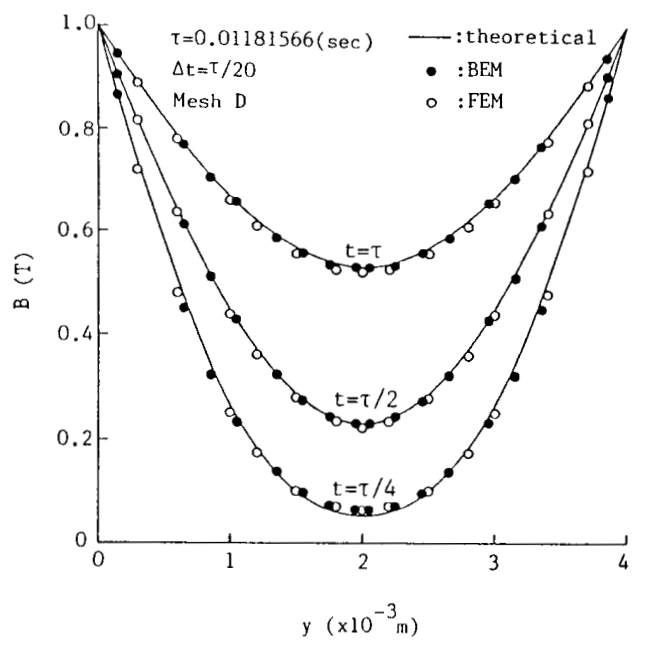

Fig. 7 Distributions of the magnetic flux density in the diffusion model.

\section{REFERENCES}

[1] C. A. Brebbia and S. Walker, Boundary Element Techniques in Engineering, London: Butterworth, 1980, ch. 6, pp.147-149.

[2] M. P. Krefta and 0. Wasynczuk, "A Finite Element Based State Model of Solid Rotor Synchronous Machines," IEEE Transactions on Energy Conversion, Vol. EC-2, pp.21-30, March 1987.

[3] Y.Saito et a1, "Dynamic Magnetic Field Computation Using Locally Orthogonal Discretization," Digests of the Intermag Conference, No. CH-0l, Tokyo, Japan, Apri1 14-17, 1987.

[4] A. Krawczyk and J. Turowski, "Recent Development in Eddy Current Analysis," IEEE Transactions on Magnetics, Vol. MAG-23, pp.3032-3037, September 1987

[5] A. Krawczyk, "The Application of the Boundary Element Method to the Transient Eddy Current Analysis," Digests of the Intermag Conference, No. GF-10, Tokyo, Japan, Apri1 14-17, 1987. 